\title{
Interactive comment on "The role of Arctic sea ice loss in projected polar vortex changes" by Marlene Kretschmer et al.
}

\section{Anonymous Referee \#1}

Received and published: 2 August 2020

Authors attempted to investigate the role of Barents-Kara (BK) sea ice loss in the future on stratospheric polar vortex (SPV) change. The BK sea ice loos at the moment appears to weaken the SPV on the observation (mainly based on reanalysis data), but whether this relation is due to global warming (i.e. forced response) or internal variability remains unclear. Some studies have suggested that the influence of the Arctic warming on mid-latitude weather may not exist or ends soon. Other studies suggested that a change in atmospheric circulation drives sea ice over the BK rather than the other way around. This study contributes to the clarification of the influence of BK sea ice loss on SPV and shows causal relation between the BK sea ice change and others. Though model responses are small to future loss of Sea ice and responds variously, ensemble mean suggests some meaning for the change in SPV, which has nonlinear 
responses to the degree of global warming or BK sea ice loss. Overall, I recommend for publication in the journal WCD with minor revisions.

WCDD

1. The relation between NP SLP to Ural SLP shows slight negative relation. What does this mean?

2. Currently, November BK SIC -> January Ural SLP is provides. How about other

Interactive

comment combinations like D BK SIC -> J Ural SLP, etc.

3. V'T' vs. BK SIC change or year (or degree of warming) would provide a good measure to determine why SPV has such nonlinear responses.

4. Models suggests that under large global warming, SPV will be enhanced. What would the possible factors for strengthening the future SPV.

5. Please fix line 246, Fig. 4c -> Fig. 4b, Fig. 4d-> Fig. 4c, and line 258, Fig. 4e -> Fig. 4d

Interactive comment on Weather Clim. Dynam. Discuss., https://doi.org/10.5194/wcd-2020-29, 2020. 